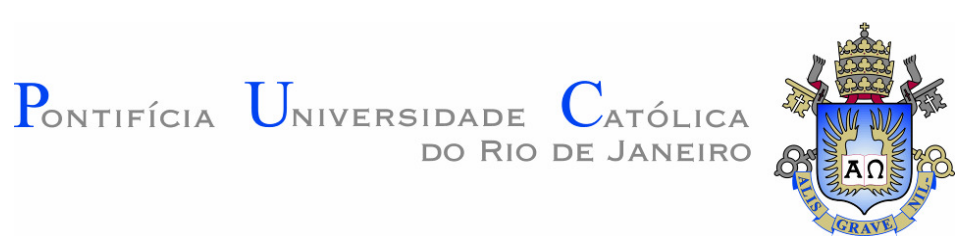

Víctor Raúl Guillén Núñez

\title{
Análise do Processo de Recuperação de Óleo por Injeção de Emulsão
}

\section{Tese de Doutorado}

Tese apresentada ao Programa de Pós-Graduação em Engenharia Mecânica do Departamento de Engenharia mecânica da PUC - Rio como requisito parcial para obtenção do título de Doutor em Engenharia Mecânica.

Orientador: Prof. Márcio da Silveira Carvalho Co-Orientador: Prof. Vladimir Alvarado Basante 


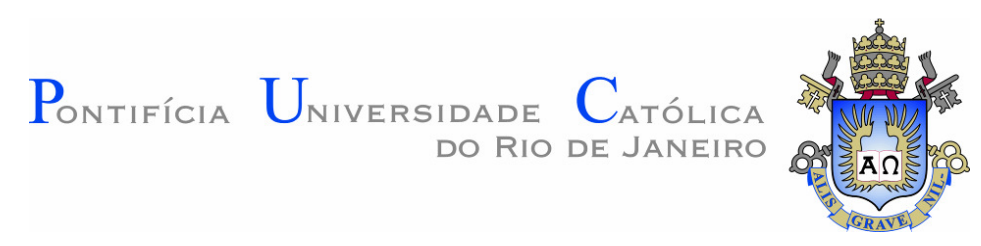

Víctor Raúl Guillén Núñez

\section{Análise do Processo de Recuperação de Óleo por Injeção de Emulsão}

Tese apresentada como requisito parcial para obtenção do grau de Doutor pelo Programa de Pós-Graduação em Engenharia Mecânica da PUC-Rio. Aprovada pela Comissão Examinadora abaixo assinada.

Prof. Márcio da Silveira Carvalho Orientador Departamento de Engenharia Mecânica - PUC-Rio

Prof. Vladimir Alvarado Basante Co-Orientador University of Wyoming

Prof. Luis Fernando Azevedo Departamento de Engenharia Mecânica - PUC-Rio

Prof. Eurípedes do Amaral Vargas Departamento de Engenharia Civil - PUC-Rio

Prof. Antonio Luiz Serra de Souza

Petrobras

Prof. Roney Leon Thompson

Departamento de Engenharia Mecânica - UFF

Prof. José Eugenio Leal

Coordinador Setorial do Centro Técnico Científico - PUC-Rio

Rio de Janeiro, 12 de Agosto de 2011. 
Todos os direitos reservados. É proibida a reprodução total ou parcial do trabalho sem autorização da universidade, do autor e do orientador.

\section{Víctor Raúl Guillén Núñez}

Graduou-se em Engenharia Mecânica na Universidad Católica de Santa Maria - UCSM (Arequipa, Perú). Fez Mestrado na PUC-Rio (Rio de Janeiro, Brasil), na área de Petróleo e Energia, estudou o Deslocamento de Óleo em um Meios Poroso Através de Injeção de Emulsões Óleo-emÁgua: Análise de Fluxo Linear.

Ficha Catalográfica

Guillén Núñez, Víctor Raúl.

Análise do Processo de Recuperação de Óleo por Injeção de Emulsões / Víctor Raúl Guillén Núñez; orientador: Márcio da Silveira Carvalho. - 2011; co-orientador: Vladimir Alvarado Basante.

v., 119 f.: il.(color).; $30 \mathrm{~cm}$

Tese (Doutorado em Engenharia Mecânica)-Pontifícia Universidade Católica do Rio de Janeiro, Rio de Janeiro, 2011.

Incluí bibliografia.

1. Engenharia Mecânica - Teses. 2. Emulsão;. 3. Controle de mobilidade;. Recuperação Avançada;. 4. Simulação de Reservatórios. I. Márcio da Silveira Carvalho. II. Vladimir Alvarado Basante. III. Pontifícia Universidade Católica de Rio de Janeiro. Departamento de Engenharia Mecânica. IV. Título.

CDD: 621 


\section{Agradecimentos}

A Deus por estar sempre comigo.

A minha família.

Aos professores Márcio da Silveira Carvalho e Vladimir Alvarado Basante, pela orientação, estímulo e confiança depositada em mim, durante nosso percurso investigativo, que permitiram a conclusão deste trabalho.

Ao Departamento de Engenharia Mecânica da Pontifícia Universidade Católica de Rio de Janeiro PUC-Rio, pela bolsa de isenção, seus professores e funcionários, pela acolhida durante o doutorado.

À FAPERJ, pelo auxílio financeiro concedido durante o curso.

Aos membros da banca examinadora, que aceitaram revisar o trabalho e contribuíram com valiosas sugestões e observações.

Aos amigos e colegas do grupo de trabalho do professor Márcio Carvalho e do Laboratório de Micro-hidrodinâmica e Escoamento em Meios Porosos LMMP pela sua ajuda direta ou indireta na realização deste trabalho.

A meus caros amigos Gerardo Castillo, Marco Rosas, Cristian Mejia, Jorge Hinostroza, e especialmente a minha querida Jaci. 


\section{Resumo}

Guillén Núñez, Víctor Raúl; Carvalho, Márcio da Silveira; Alvarado Basante, Vladimir. Análise do Processo de Recuperação de Óleo por Injeção de Emulsões. Rio de Janeiro, 2012. 119p. Tese de Doutorado Departamento de Engenharia Mecânica, Pontifícia Universidade Católica do Rio de Janeiro.

A injeção de água é o método mais comum para manter a pressão e melhorar a recuperação de óleo contido em um reservatório. A eficiência de recuperação de óleo no caso de óleos pesados é limitada pela alta razão de mobilidade entre o fluido deslocante e o fluido deslocado. Como a sede mundial por energia aumenta todo ano, enquanto o fluxo de petróleo dos campos petrolíferos conhecidos juntamente com a descoberta de novos reservatórios declina a uma velocidade considerável, torna-se indispensável utilizar métodos mais efetivos para extrair o petróleo dos reservatórios conhecidos. Diferentes métodos de recuperação avançada de óleo são desenvolvidos em busca de alternativas. A injeção de dispersões, em particular a injeção de emulsões óleoem-água, como um agente de controle de mobilidade do fluido injetado tem sido testada e estudada com relativo sucesso. Porem esta técnica ainda não é totalmente desenvolvida ou compreendida. O uso efetivo de injeção de emulsões como uma alternativa para a recuperação de petróleo requer uma completa análise dos diferentes regimes de fluxo de emulsões dentro do espaço poroso de um reservatório. Se o tamanho de gota da fase dispersa for da mesma ordem de magnitude do tamanho de poro, as gotículas podem se aglomerar e bloquear parcialmente o escoamento através do espaço poroso, controlando assim a mobilidade do fluido deslocante, obtendo assim um deslocamento mais uniforme e um aumento no fator de recuperação. Este trabalho tem como objetivo principal o estudo do processo de deslocamento de óleo em um meio poroso por injeção de água e emulsões óleo-in-água. Diferentes experimentos foram realizados para análise de diferentes aspectos do problema, incluindo a injeção alternada de água 
e emulsão óleo-em-água a diferentes vazões, injeção alternada de água e emulsão em meios com diferentes permeabilidades conectados paralelamente e visualização do escoamento através de um meio poroso transparente formado por esferas de vidro não consolidadas. Um modelo do escoamento de emulsão foi considerado através da modificação da curva de permeabilidade relativa da fase aquosa, que é escrita como função não só da saturação, mas também da concentração de gotas de emulsão e do número de capilaridade local. O processo de deslocamento de óleo através de injeção alternada água-emulsão foi também estudado numericamente através de um código desenvolvido em Matlab utilizando o modelo TPFA (Two Flux Approximation) and IMPES (IMplicit Pressure and Explicit Pressure Saturation).

\section{Palavras-chave}

Emulsão; controle de mobilidade; recuperação avançada de petróleo; simulação de reservatório; meio poroso. 


\section{Abstract}

Guillén Núñez, Víctor Raúl; Carvalho, Márcio da Silveira (Advisor); Alvarado Basante, Vladimir (co-Advisor). Analysis of Oil Recovery Process by Emulsion Injection. Rio de Janeiro 2012. 119p. Doctoral Thesis - Departamento de Engenharia Mecânica, Pontifícia Universidade Católica de Rio de Janeiro.

Water injection is a common method to maintain reservoir pressure and improve oil recovery. The efficiency of oil recovery in the case of heavy oils is limited by the high mobility ratio between the injected water and oil. As the world thirst for energy is increasing every year while oil production from known oil reservoirs together with the discovery of new oil reservoirs deplete at considerable rate, it becomes indispensable to use more effective methods to produce oil from known reservoirs. The injection of dispersions, in particular of oil-in-water emulsions, as an agent of mobility control of injected fluid has been tested and studied with relative success. However this technique is not completely developed and understood. The effective use of emulsion injection as an alternative for oil recovery needs a complete analysis of different regimes of emulsion flow through the pore space of a reservoir. If the drop size of the dispersed phase is of the same order of magnitude of the pore size or lager, the drops can agglomerate and partially block the flow through the pores, thus controlling the displacing fluid mobility, getting a more uniform displacing front and an increase in the oil recovery factor. The main goal of this work is the study of oil displacement process through a porous media by water and oil-in-water emulsion injection. Different experiments were carried out for analysis of different aspects of the problem, including the alternating injection of water and oil-in-water emulsion at different flow rates, through cores with different permeabilities connected in parallel, and visualization of flow through a transparent non consolidated porous media, formed by glass beads. A model of emulsion flow was considered by modifying the relative permeability curve of the aqueous phase, which is written 
as a function not only of the aqueous phase saturation, but also as a function of the emulsion drop concentration and local capillarity number. The process of oil displacement by alternated water-emulsion injection was also studied numerically by a code developed in Matlab using TPFA (Two Flux Approximation) and IMPES (IMplicit Pressure and Explicit Pressure Saturation) methods.

\section{Keywords}

Emulsions; mobility control; enhanced oil recovery; reservoir simulation; porous media. 


\section{Sumário}

1 Introdução 13

1.1 Motivação 15

1.2 Objetivo 22

1.3 Escopo 22

2 Conceitos Fundamentais 23

2.1 Porosidade 23

2.2 Molhabilidade $\quad 24$

2.3 Pressão Capilar 25

2.4 Permeabilidade $\quad 27$

2.4.1 Curvas de Permeabilidade Relativa 28

2.5 Numero de Capilaridade $\quad 29$

2.6 Mobilidade 30

$\begin{array}{ll}\text { 2.7 Revisão Bibliográfica } & 31\end{array}$

3 Metodologia Experimental $\quad 35$

3.1 Descrição experimental do dispositivo para visualização do escoamento de emulsões através do meio poroso 35

3.1.1 Bomba de injeção $\quad 37$

3.1.2 Mecanismo cilindro-pistão 38

3.1.3 Câmara portadora do meio poroso transparente não consolidado 38

3.1.4 Meio poroso 38

3.1.5 Transdutor de pressão $\quad 39$

3.1.6 Aquisição e armazenamento de dados $\quad 39$

3.1.7 Balança eletrônica $\quad 40$

3.1.8 Fluidos utilizados no experimento visualização do escoamento de emulsões através do meio poroso 40

3.2 Descrição do dispositivo experimental para estudo do efeito do numero de capilaridade no processo de deslocamento de óleo. 42

3.2.1 Bomba de injeção 43

3.2.2 Mecanismo cilindro-pistão $\quad 44$

3.2.3 Câmara porta-amostra tipo Hassler 44

3.2.4 Corpos de prova $\quad 45$

3.2.5 Balança eletrônica 46

3.2.6 Fluidos utilizados no experimento de capilaridade 46

3.3 Procedimento experimental para estudo do escoamento em amostras de rocha em paralelo 46

$\begin{array}{ll}\text { 3.3.1 Bomba de injeção } & 47\end{array}$

$\begin{array}{ll}\text { 3.3.2 Mecanismo cilindro-pistão } & 47\end{array}$ 
3.3.3 Câmara porta-amostra tipo Hassler 47

$\begin{array}{ll}3.3 .4 \text { Corpo de prova } & 48\end{array}$

$\begin{array}{ll}\text { 3.3.5 Transdutores de pressão } & 49\end{array}$

3.3.6 Aquisição e armazenamento de dados 49

3.3.7 Balança eletrônica $\quad 49$

3.3.8 Fluidos utilizados no experimento de amostras em paralelo 49

3.4 Metodologia Utilizada para Caracterizar os Meios Porosos e os Fluidos Utilizados $\quad 50$

3.4.1 Caracterização dos Meios porosos $\quad 50$

3.4.2 Caracterização dos líquidos $\quad 54$

4 Resultados Experimentais $\quad 57$

4.1 Visualização do escoamento de emulsões através do meio poroso. $\quad 57$

4.1.1 Resultados \# 1

4.1.2 Resultados \# 2

4.1.3 Resultados \# 3

4.2 Efeito do numero de capilaridade no processo de deslocamento de óleo $\begin{array}{ll}\text { por injeção de emulsões em meios porosos } & 67\end{array}$

4.3 Efeito da injeção de emulsões no deslocamento de óleo de duas amostras de arenito com diferentes permeabilidades colocadas 71

4.4 Analise da Emulsão Produzida $\quad 74$

5 Modelagem Numérica $\quad 77$

$\begin{array}{ll}5.1 \text { Formulação Matemática } & 77\end{array}$

5.1.1 Escoamento Monofásico $\quad 77$

$\begin{array}{ll}5.1 .2 \text { Escoamento Multifásico } & 78\end{array}$

$\begin{array}{ll}5.1 .3 \text { Injeção de Emulsões } & 81\end{array}$

5.1.4 Discretização do Problema 83

5.2 Modelo Numérico $\quad 85$

5.2.1 Estratégias de Solução para um Sistema Acoplado 85

5.2.2 Discretização da Equação de Pressão 86

5.2.3 Discretização da Equação de Saturação 88

5.2.4 Discretização da Equação de Saturação de Emulsão 89

5.2.5 Método IMPES 90

5.3 Implementação 93

5.4 Resultados Numéricos. 95

$\begin{array}{ll}\text { 5.4.1 Testes preliminares } & 96\end{array}$

5.4.2 Bifásico com Emulsão 97

6 Comentários finais e sugestões $\quad 113$

6.1 Comentários finais 113

Referencias Bibliográficas $\quad 115$ 


\section{Lista de figuras}

1.1 Estrutura esquemática de um reservatório de petróleo, tipo anticlinal. 13

1.2 (a) Aoramento de arenito de grãos de areia, 14

1.3 Classificação dos métodos de recuperação Avançada [1]. 18

1.4 Fotografia de uma emulsão óleo em água, tomada com microscópio 20

1.5 Tipos de emulsões. 20

1.6 Aglomeração e bloqueio de poros com gotas de emulsão. 21

2.1 Matriz rochosa e espaço poroso de um corpo. 23

2.2 Equilíbrio de forcas na interface óleo água. 24

2.3 Molhabilidade em sistemas óleo água. 25

2.4 Elevação de água em um tubo capilar. 26

2.5 Curvas de pressão capilar na drenagem e embebição. 27

2.6 Fluxo linear em um meio poroso horizontal. 28

2.7 Curvas típicas de permeabilidade relativa de rocha molhada por água. 29

2.8 Efeito da variação da saturação sob a permeabilidade relativa de um sistema água óleo. 30

3.1 Esquema do dispositivo experimental de visualização do escoamento de emulsões através do meio poroso 36

3.2 Fotografia da câmara portadora do meio poroso transparente não consolidado. 39

3.3 Representação esquemática do dispositivo experimental para o estudo do efeito do numero de capilaridade no processo de deslocamento 42

3.4 Fotografia do dispositivo experimental para o estudo do efeito do numero de capilaridade no processo de deslocamento de óleo.

3.5 Esquema mostrando os componentes da câmara portadora de amostras tipo Hassler. 45

3.6 A amostra de arenito. $\quad 45$

3.7 Representação esquemática do dispositivo experimental para o estudo do escoamento em amostras de rocha em paralelo.

3.8 Esquema mostrando os componentes da câmara portadora de amostras.

3.9 Fotografia do método utilizado para embeber o corpo de prova 51

3.10 Curva de Vazão Diferencial de Pressão. 53

3.11 Fotografia de uma emulsão mostrando alguns diâmetros das gotas 
da emulsão D2 $=20 \mu \mathrm{m}$.

4.1 Fator de Recuperação mediante injeção alternada de água e emulsão $\mathrm{O} / \mathrm{W}$, em um meio poroso de vidro.

4.2 Fator de Recuperação mediante injeção alternada de água e emulsão O/W, em um meio poroso de vidro.

4.3 Fotografias mostrando o segundo processo de Recuperação mediante injeção alternada de água e emulsão o/a, em um meio poroso

4.4 Fotografias mostrando o processo aglomeração das gotas de emulsão o/a, em um meio poroso de vidro.

4.5 Visualização da mudança do caminho preferencial dos fluidos

4.6 Fotografia mostrando o caminho percorrido pelos fluidos injetados no inicio do experimento.

4.7 Fotografia mostrando o caminho percorrido pelos fluidos injetados no final do experimento.

4.8 Resultado da recuperação de óleo de uma amostras de arenito mediante a injeção de água e emulsão óleo em água

4.9 Resultado da recuperação de óleo de uma amostras de arenito mediante a injeção de água e emulsão óleo em água a numero de capilaridade alto.

4.10 Resultado do primeiro experimento de injeção de emulsões no deslocamento de óleo de duas amostras de arenito com diferentes permeabilidades colocadas em paralelo.

4.11 Resultado do segundo experimento de injeção de emulsões no deslocamento de óleo de duas amostras de arenito com diferentes permeabilidades colocadas em paralelo.

4.12 Distribuição do tamanho de gota da emulsão antes e depois do processo de deslocamento de óleo.

4.13 Fotos de fluidos produzidos do processo de deslocamento de óleo.

5.1 Representação da redução da permeabilidade relativa em função da saturação de água, devido a concentração de emulsão. 